\title{
Caecal Bascule: A Rare Complication Following Caesarean Section
}

\author{
Amira Salih ${ }^{\mathrm{a}}$, Junaid Rafi, ${ }^{\mathrm{a}}$, Elnazeer Salim ${ }^{\mathrm{b}}$
}

\begin{abstract}
We present a rare case of caecal bascule in a forty year old Caucasian, multiparous lady ( 3 previous normal vaginal deliveries and one caesarean section) who had a grade 1 (fetal distress) uneventful caesarean section $(\mathrm{C} / \mathrm{S})$ under general anaesthesia. On the third post-operative day, she developed marked abdominal distension and pain but no vomiting. Abdominal examination showed a tender palpable tympanic mass in the right upper quadrant and epigastrium. Caecal volvulus was suspected and confirmed on radiological investigations. Emergency laparotomy was performed. Intra-operative findings showed rotation of the caecum over itself resulting in a sharp twist on the ascending colon with patches of necrosis and serosal tears of the caecum. The ascending colon and terminal ileum were normal. These findings are classical description of "Caecal Bascule". Limited right hemi-colectomy was performed. The patient made uneventful speedy recovery.
\end{abstract}

Keywords: Caecal bascule; Caecal volvulus

\section{Introduction}

Mechanical intestinal obstruction is a rare but serious complication following caesarean section with reported incidence of 1 in 1,500 to 1 in 66,431 [1]. Volvulus is responsible for $24 \%$ of intestinal obstruction during pregnancy and puerperium. Caecal volvulus (CV) is an axial twist of caecum, terminal ileum and ascending colon. Caecal bascule is a rare form of

\footnotetext{
Manuscript accepted for publication July 1, 2010

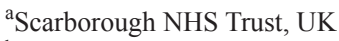

${ }^{\mathrm{b}} \mathrm{Hull}$ and East Yorkshire NHS Trust, UK

${ }^{\mathrm{c} C o r r e s p o n d i n g ~ a u t h o r: ~ S c a r b o r o u g h ~ N H S ~ T r u s t, ~ U K . ~}$

Email: drjunaidrafi@hotmail.com
}

doi:10.4021/jmc2010.07.110e volvulus in which there is an upward folding of the caecum on itself producing a sharp kink in the ascending colon [2].

\section{Case Report}

A forty year old Caucasian, multiparous lady presented posterm in spontaneous labour. She had 3 previous normal vaginal deliveries and one caesarean section. She was for trial of scar, however, due to fetal distress a grade 1 uneventful caesarean section $(\mathrm{C} / \mathrm{S})$ was performed under general anaesthesia. On the third post-operative day, she developed marked abdominal distension and pain but no vomiting. Abdominal examination showed a tender palpable tympanic mass in the right upper quadrant and epigastrium.

Caecal volvulus was suspected. Abdominal X-ray (Fig. 1) supported the diagnosis and CT scan revealed that the caecum was hugely distended $(12 \mathrm{~cm})$, although there was no clear cut off point. Prompt surgical review raised the suspicion of impending caecal perforation. Rectal examination and rigid sigmoidoscopy showed collapsed rectum which made the possibility of pseudo-obstruction less likely. Emergency laparotomy was performed. Intra-operative findings showed rotation of the caecum over itself resulting in a sharp twist on the ascending colon with patches of necrosis and se-

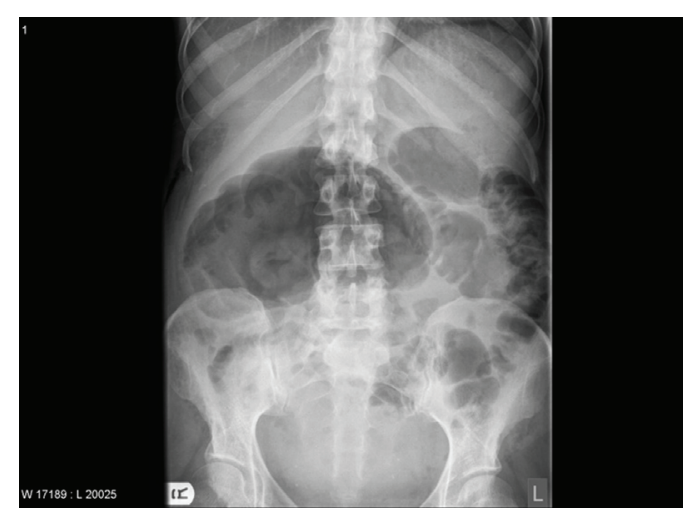

Figure 1. Plain abdominal X-Ray shows hugely distended caecum. 
rosal tears of the caecum. The ascending colon and terminal ileum were normal. These findings are classical description of "Caecal Bascule". Limited right hemi-colectomy was performed. The patient made uneventful speedy recovery.

\section{Discussion}

Intestinal obstruction complicating pregnancy is rare with reported incidence of $1: 1,500$ to $1: 66,431$. In general colonic volvulus represents $24 \%$ of the cases [1]. Regarding post caesarean section caecal volvulus in particular there have been only 10 cases reported in the English literature untill 1980 [3], one in 1988 [4], and only three cases from 2000-2006 [5-7], while the earliest case report available is reported by Tumacder et al in 1964 [8]. To our best knowledge this is the first case reported in English medical literature about caecal bascule which is a rare form of volvulus in which there is an upward folding of the caecum on itself producing sharp kink in the ascending colon [2].

During pregnancy the gravid uterus displaces the caecum upwards, and after caesarean section there is sudden change of intra-abdominal pressure. Both are predisposing factors for caecal volvulus.

Presentation of volulus is uncommon in adulthood and recognition during pregnancy and puerperium is often delayed, which can lead to serious morbidity and mortality. Reports on caecal volvulus mortality rate show a $12 \%$ rate in patients with viable caecum compared to $33 \%$ if the caecum was gangrenous [9]. The usual presentation of distension and pain can mimic more familiar conditions after cesarean section such as paralytic ileus and pseudo-obstruction. Paralytic ileus is common following cesarean sections complicated by postpartum hemorrhage and repeat Cesarean Section with adhesions [10]. High index of suspicion is a key factor to early correct diagnosis.

The classical radiological features of caecal volvulus are: comma-shaped caecal shadow in mid-abdomen or to the left upper quadrant in supine abdominal X-Ray and single long fluid level on erect film.

The treatment is surgical, and laparotomy is necessary in most patients. Right hemi -colectomy is the treatment of choice. It eliminates all risks or recurrence and has small risk of leak. Other procedures, if the caecum is viable, are caecostomy and caecopexy. However there are associated risks of abdominal wall sepsis, fasciitis, recurrence (0-25\%) and mortality (0-33\%) [11].

It demonstrates the importance of having an open mind when evaluating obstetrics patients with common symptom of pain and abdominal distension. Such symptoms might be dismissed or attributed to usual obstetrics disorders.

In conclusion, early involvement of consultants and multidisciplinary approach including liaison with surgeons, radiologists and urgent access to investigations out of hours was exemplified in this case. With a steady rise of cesarean section rate reaching an incidence of $24.3 \%$, trainees will need to be more vigilant and extend their awareness to even rare complications of commonly performed procedures [12] .High index of suspicion of such a rare but serious complication in puerperal period is crucial to accurate diagnosis and prompt management if serious morbidity and mortality is to be avoided.

\section{Poster Presentation}

Salih A, Mumdjzans A, Jha R, Salim E .Caecal Bascule: A Rare Complication Following Caesarean Section. Obstetrics and Gynaecology Annual Regional Conference Day in Leeds, United Kingdom on 29th Jan 2010; (Page 37).

\section{References}

1. Perdue PW, Johnson HW, Jr., Stafford PW. Intestinal obstruction complicating pregnancy. Am J Surg 1992;164(4):384-388.

2. Weinstein M. Volvulus of the caecum and ascending colon. Ann Surg 1938;107: 248-59.

3. Victor A ,Peter F H ,Demetrios Y,Patrick V.Caecal volulus following Cesarean section. Obstet Gynecol 1980;55:131-34.

4. Fanning J, Cross CB. Post-cesarean section cecal volvulus. Am J Obstet Gynecol 1988;158(5):1200-1202.

5. Al Salamah SM, El Keyali AY. Ileo-caecal volvulus post-cesarean section: a case report. Saudi J Gastroenterol 2000;6(3):163-164.

6. Pal A, Corbett E, Mahadevan N. Caecal volvulus secondary to malrotation presenting after caesarean section. J Obstet Gynaecol 2005;25(8):805-806.

7. Marren A, Wong K. Caecal volvulus associated with intestinal malrotation immediately following caesarean section. N Z Med J 2006;119(1240):U2130.

8. Tumacder OC, Reynolds RM. Cecal Volvulus Following Cesarean Section. Grace Hosp Bul 1964;42(79-82.

9. Ballantyne $\mathrm{GH}$, Brandner MD, Beart RW, Jr., Ilstrup DM. Volvulus of the colon. Incidence and mortality. Ann Surg 1985;202(1):83-92.

10. Green top guide line 52: Prevention and management of post partum haemorrhage. RCOG, May 2009.

11. Abbasakoor F and Vaizey C. Colonic emergencies. In A Companion to Specialist Surgical Practice. Core Topic in General and Emergency Surgery. Simon PatersonBrown. Third Edition 2005.

12. NHS Maternity Statistics, England 06-07 Published 25th Sep 2008. 